\title{
A Finite Element Method for Deformable Models
}

\author{
Persephoni Karaolani, G.D. Sullivan, K.D. Baker \& M.J. Baines \\ Intelligent Systems Group, Department of Computer Science \\ University of Reading, RG6 2AX, UK, \\ P.Karaolani@reading.ac.uk
}

Deformable models of elastic structures have been proposed for use in image analysis. Previous work has used a variational approach, based on the EulerLagrange theory. In this paper an alternative mathematical treatment is introduced, based on a direct minimisation of the underlying energy integral using the Finite Element Method. The method is outlined and demonstrated, and its principal advantages for modelbased image interpretation are explained.

\section{INTRODUCTION}

Models allow the use of high-level knowledge about expected structures to control the visual interpretation process. Most work on model-based vision has considered rigid [1] or parameterised [2] models. Recently, deformable models have also attracted attention, e.g. [3,4,5]. Deformable models are likely to have particular relevance in medical imaging applications, as a means of encoding the shapes of anatomical organs, which are inherently variable.

As an example, consider an X-ray image of an artery. Context-free image processing may produce good evidence of an artery in the regions A and B (see Figure. 4(a)), but fail to identify its position between $A$ and $B$. One way to detect the artery in this space is to use a model of an "ideal" artery, which can be deformed within known constraints under the influence of the fragmentary image data, to derive an optimal global solution.

A simple artery model can be specified by minimising the energy integral given in expression (1) over functions v(s), as proposed by Kass et. al. [3].

$$
\int_{0}^{1}\left\{\alpha(s)\left|v_{s}(s)\right|^{2}+\beta(s)\left|v_{s s}(s)\right|^{2}+I(v(s))\right\} d s
$$

Where $v(s)=(x(s), y(s))$ is the position vector as a function of the intrinsic parameter $s$, the normalized length of the line with vertices $v_{1}, v_{2} \ldots v_{M}$ [note: $v_{i}$ $(i=1 \ldots . M)$ denotes $\left.v\left(s_{i}\right)\right]$. The terms $v_{s}$ and $v_{s s}$ denote first and second derivatives of $\mathrm{v}$ with respect to $\mathrm{s}$; and $\alpha(s), \beta(s)$ are weighting functions.

Expression (1) contains three terms, the minimisation of which have simple physical interpretations.
- The term $\left|v_{s}(s)\right|^{2}$ (the norm of the first derivative of the position vector) makes the model act like an elastic string.

- The term $\left|v_{s s}(s)\right|^{2}$ (the norm of the second derivative of the positional vector) makes the model act like a thin bar.

- The final term, $I(v(s))$, is used to allow chosen features of the image intensity values to influence the model. In the present treatment we use the image grey-values along $v(s)$, so that the term is minimised in dark areas of the image.

In effect, the minimisation of expression (1) expresses the fact that an artery is a stiff, elastic, dark entity. The optimal global solution of the position of an artery is the choice of $v(s)$ which minimises the total energy given in (1). In general this is a non-linear problem which must be solved using iterative numerical methods, which produce successive deformations of the position $\mathrm{v}(\mathrm{s})$.

A method for minimising integral (1) based on the use of the Euler-Lagrange Theory (ELT) has been presented previously $[3,4,5]$. This paper presents an alternative approach based on the use of the Finite Element Method (FEM). In recent years, FEM has been used extensively in computational studies of elasticity in structural mechanics. It offers benefits over the more traditional ELT, which it has now superceded in many areas of study. We report here a modification to the FEM which allows it to be used for model-based vision, and discuss its computational advantages over ELT.

\section{ENERGY MINIMISATION BY ELT \& FEM}

Both ELT and FEM may be used to solve variational problems, including the minimisation of energy integrals such as expression (1). The principles of both methods have been treated extensively in the literature [6,7]. The two approaches are illustrated in Figure 1, and their main characteristics are outlined briefly below.

\section{(i) Euler-Lagrange Theory}

The ELT solves a variational problem by reducing it to the solution of differential equations. The ELT method used by $[3,4,5]$ develops a set of differential equations by considering the change in the energy integral caused by small variations in each of the unknowns $x(s), y(s)$. 


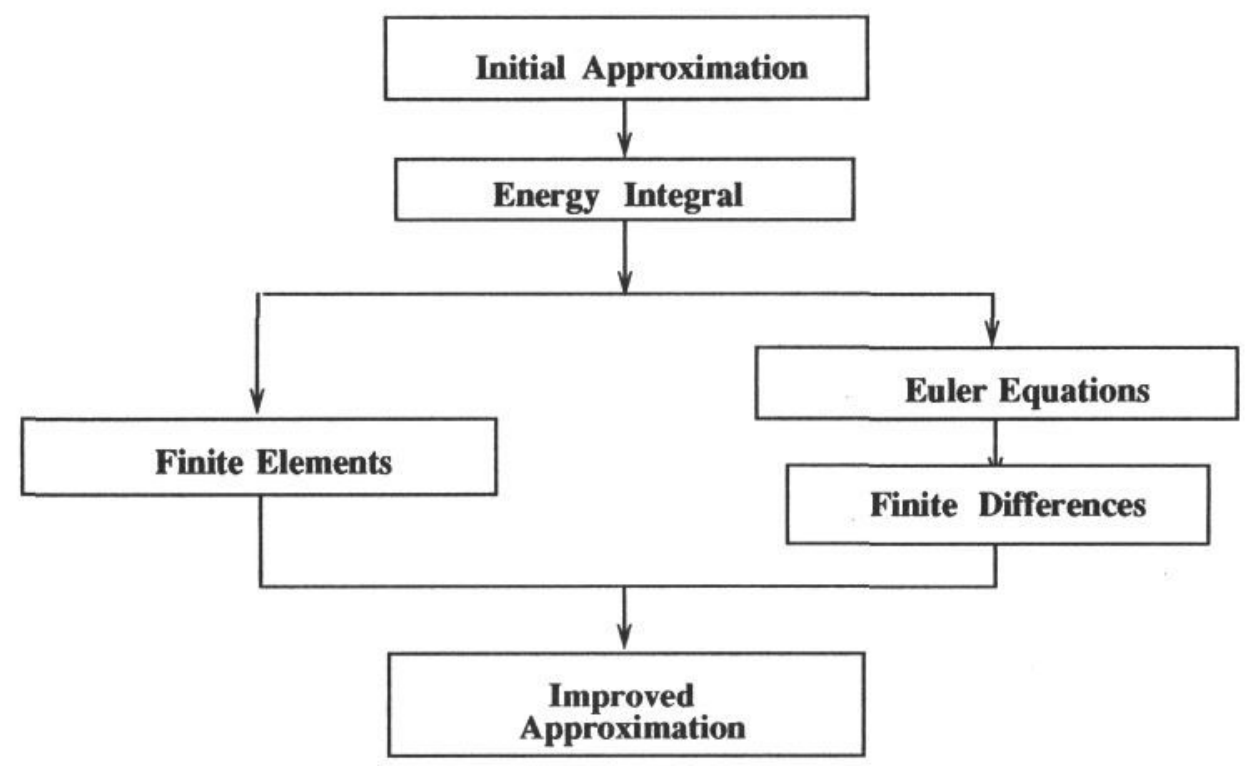

Figure 1: Schematic showing alternative approaches to energy minimisation, using FEM (left) or ELT (right). FEM is more direct since it involves a single stage of approximation, whereas ELM requires two

This gives rise to the Euler-Lagrange equations. These higher-order differential equations are then made discrete and solved numerically by means of finite differences.

\section{(ii) Finite Element Method}

FEM uses the energy integral directly, and works by dividing the given domain into subdomains and minimising the energy integral over each subdomain separately. This produces the so called "element equations" which are then joined together through their common boundaries.

External forces which are independent of the unknowns to be determined may be represented in FEM as a set of loading conditions. FEM also leads to a system of equations, the solution of which involves a matrix. In this case the matrix is not only banded, but is also symmetric and positive-definite.

\section{The advantages of FEM over ELT}

A main advantage of the FEM in minimising energy integrals such as expression (1) is that the method is more straightforward. It acts directly on the integral, instead of transforming the integral to a set of differential equations to be solved numerically (see Figure 1).

Further, there are difficulties inherent in the ELT approach, especially when several independent variables are involved (requiring partial derivatives). This constrains both the type of integral that can be minimised by ELT, and the choice of boundary conditions [8]. FEM provides a very flexible approach to minimising energy integrals, and is capable of handling a wide variety of energy integrals and boundary conditions.

ELT is also ineffective in solving problems that are geometrically complex, or whose loads or physical properties are discontinuous [9]. FEM is well-suited to this task [10].

There are a number of other advantages of FEM over the use of ELT solved by finite differences, which are of particular relevance to applications involving deformable models:

- FEM leads to matrices which are n-diagonal, symmetric, and positive-definite, for which efficient numerical schemes have been developed that use fewer operations and handle round-off errors effectively. An example of such a numerical scheme is the Cholesky decomposition [11].

The ELT leads to $n$-diagonal matrices with no guarantee of symmetry or positive-definite characteristics.

- The ability to represent domains with irregular geometries by a collection of Finite Elements makes the method a valuable practical tool for modeling regular structures. This feature has proved important for the solution of problems in structural engineering. Domains with curved boundaries can be treated very effectively by using "isoparametric" elements [12], and this is particularly useful for modeling curved surfaces by means of an elastic membrane.

By contrast, in ELT the nodes over which the derivatives are made discrete must form a rectangular mesh, which often fails to capture the underlying geometry efficiently In multiple dimensions this can lead to great inefficiency.

Figure 2 illustrates this point graphically. FEM is able to use an efficient representation of the structure, as 2 triangles and 2 quadrilaterals, which may be specified by 8 pairs of equations in 8 pairs of unknowns (corresponding to the asterisks in Figure 2(b)). On the other hand, the ELT approach (using finite differences) requires nodes spaced equally in each dimension, Figure 2(c). Very high resolution is needed to capture the shape of the structure accurately. In this case 602 -d control points are needed, giving rise to 120 unknowns.

- FEM uses a more natural description of the derivatives, able to exploit the continuity of the 


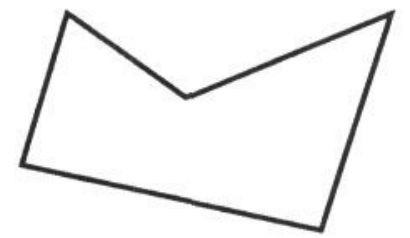

(a) Structure to be modeled

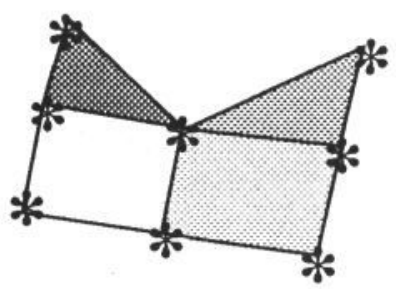

(b) Decomposition in FEM

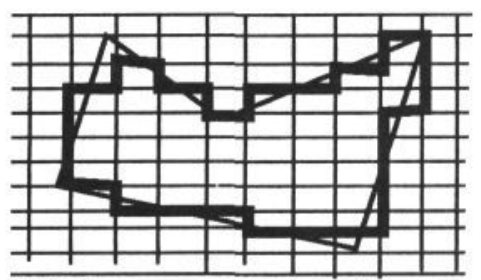

(c) Representation in ELT

(using finite differences)

Figure 2: Illustration of the advantage of using finite elements to model geometrical structures

position vector over each element.

ELT equations solved by finite differences use a discrete approximation for the derivatives.

- In the FEM we can choose between a wide selection of boundary conditions. It is also possible to impose smoothness criteria at the inter-element boundaries, with only small modifications of the final system.

ELT does not have the equivalent flexibility $[6,8]$.

\section{DEMONSTRATION OF FEM}

The use of FEM for image interpretation using deformable models has been demonstrated by means of a program which uses the same energy integral as Kass et al [3]. Details of the method are given in the Appendix. We can think of the function $v(s)$ as a "snake" which seeks out dark regions in the image.

The image term in the energy integral (1) gives rise to a set of external forces. These are calculated by estimating numerically the derivative (with respect to the unknowns, $x$ and $y$ ) of the integral of the image intensity along straight lines connecting adjacent nodes. The external forces act as the loading conditions for the system (see Appendix). As the snake moves between iterations, the image energy changes according to its position. This changes the loading conditions, which have to be re-computed on each iteration.

Our initial implementation is computationally inefficient. It has been written in pop11, and uses a
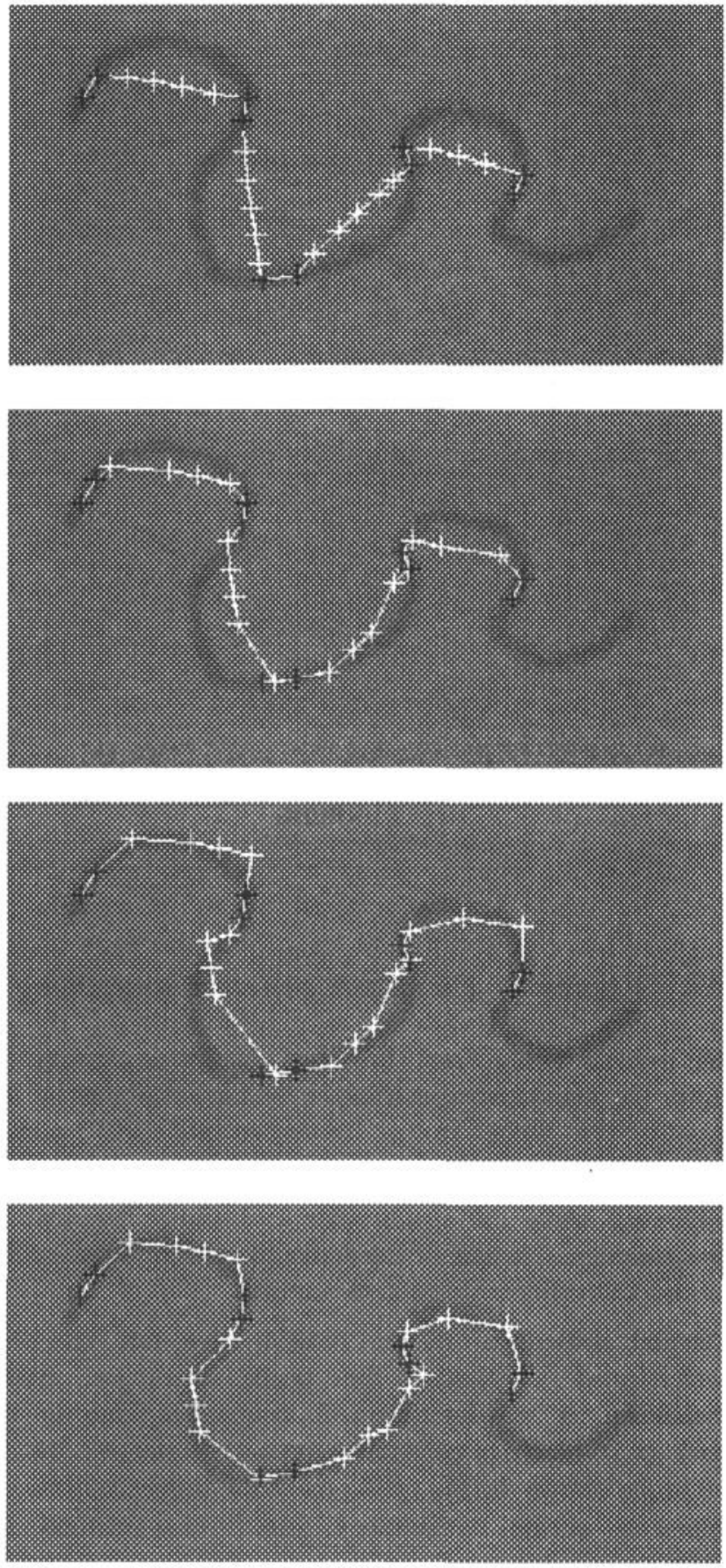

Figure 3: Demonstration of the FEM minimisation of expression (1), showing (top to bottom) the initial position, and the result after 1,3 and 9 iterations.

general-purpose matrix-inversion routine (based on the pivotal Gauss method), which has poor numerical characteristics for this problem, and fails to exploit the properties of the symmetrical, positive-definite, banded matrix. Furthermore, we have as yet employed only a very simple iterative technique. In consequence, our demonstrations are at present limited to use only a few tens of nodes, and the behaviour of the snake is correspondingly coarse. In future work we plan to make use of more efficient numerical methods.

Figure 3 shows an example of the program applied to a synthetic image. Figure 3(top) shows the initial position of the snake. The black crosses indicate fixed points, and simulate the fragmentary evidence that might 

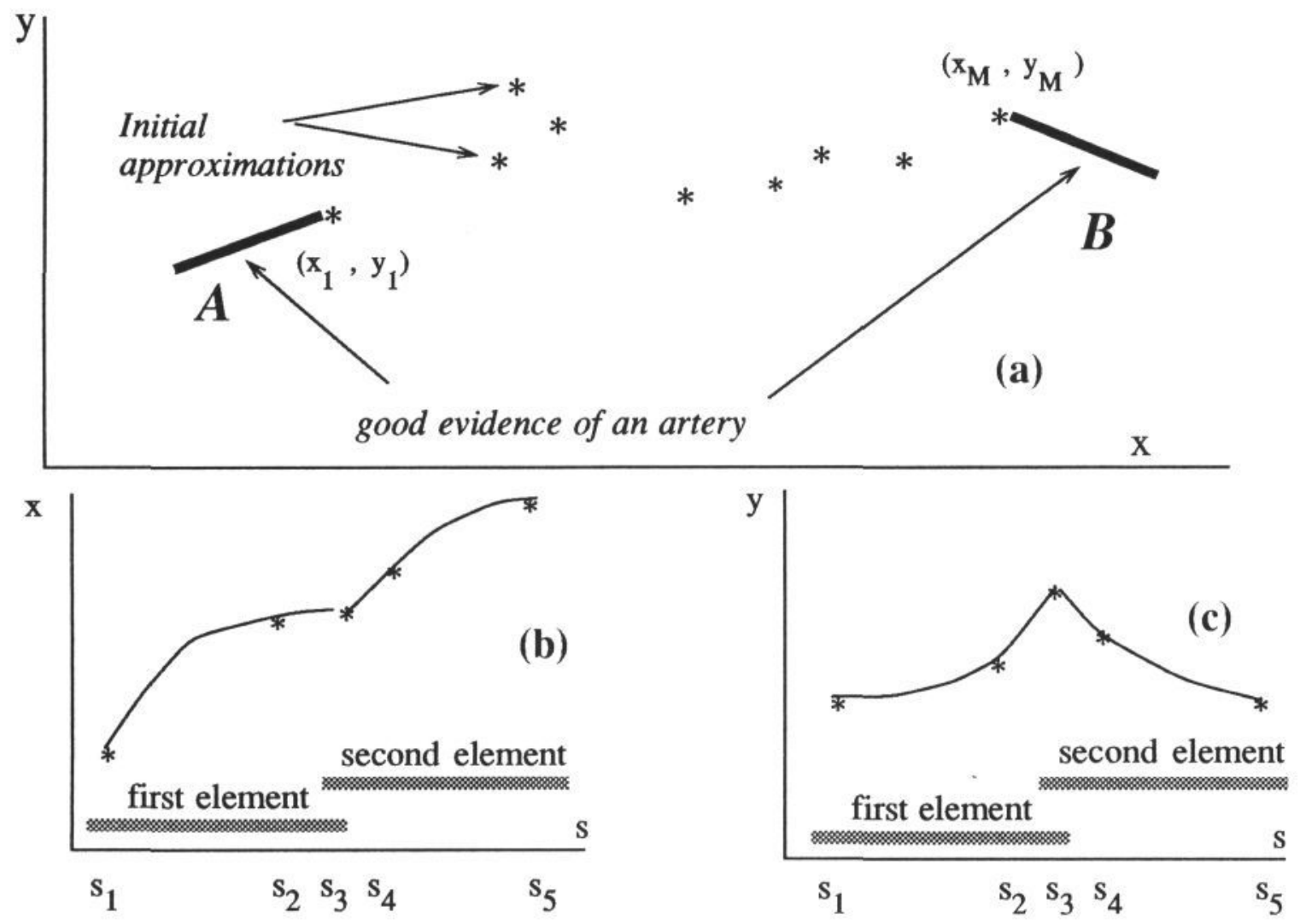

Figure 4: FE Mesh generation showing $(x, y)$ positions (top) parameterised by $s$ (bottom)

be derived either from low-level image processing, or from the interactive specification of known points by the user. The white crosses represent additional starting points interpolated between the fixed points.

Figures 3 show the results after 1, 3 and 9 iterations of the program. It can be seen that the snake successfully seeks out the linear shape in the image.

Several difficulties remain, which have not yet been investigated. Firstly, the snake manifests erratic behaviour at the inter-element boundaries, due to the lack of smoothness constraints between elements. In the example shown in Figure 3, the only influence between elements occurs where they join. One method for overcoming this has been to use over-lapping elements. This doubles the number of elements which are then combined linearly into the $\mathbf{M}^{*} \mathbf{M}$ matrix. A better alternative would be to impose first and second order continuity at the inter-element nodes.

\section{CONCLUSION}

We have presented a novel technique for using deformable models in the analysis of images, based on the finite element method. The technique has been demonstrated on a simple simulated image.

The FEM offers several important potential advantages for minimising energy expressions arising from deformable models, but these have yet to be properly investigated. In particular it offers the ability to use more complex energy terms, and may be efficiently extended to problems involving multiple dimensions. These developments are the subject of current work.

\section{APPENDIX}

\section{OUTLINE OF THE IMPLEMENTATION}

We give here a brief description of the use of FEM for a one dimensional case. The problem is to minimise the energy integral given in expression (1). We have adapted fairly standard techniques, see $[9,12]$. Section 1 below summarises conventional finite element methods, and Section 2 reports our adaptation of the method for image analysis using deformable models.

\section{(1) One Dimensional FEM}

Division of the domain into finite elements. The domain $(s)$ is divided into a finite number of elements $(m)$. Each element includes a number of nodes ( $n$ ), determined by the degree of continuity of the vector $v(s)=(x(s), y(s))$ demanded by the order of the derivatives in the energy integral. Neighbouring elements share a common node, see Figure $4(b)$, so that the total number of nodes is given by $M=1+m(n-1)$.

For the expression (1), n must be at least 3 because it includes second order derivatives of the position vector. In this case the analytical representation of the position vector $\mathrm{v}(\mathrm{s})$ must be at least quadratic over each element.

Calculation of the basis functions. The coordinates of the positional vector, $x(s)$ and $y(s)$, over each element can be expressed by:

$$
\begin{aligned}
& x(s)=\Sigma x_{j} \Phi_{j}(s) \quad(j=1 \ldots n) \\
& y(s)=\Sigma y_{j} \Phi_{j}(s) \quad(j=1 \ldots n)
\end{aligned}
$$




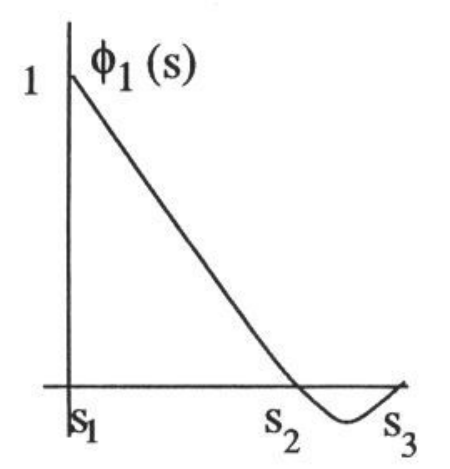

$\phi_{1}(s)=\frac{\left(s-s_{2}\right)\left(s-s_{3}\right)}{\left(s_{1}-s_{2}\right)\left(s_{1}-s_{3}\right)}$

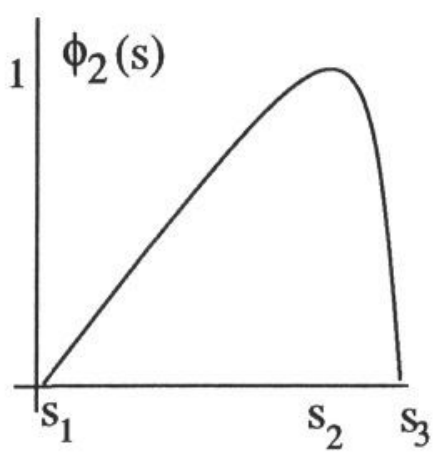

$\phi_{2}(s)=\frac{\left(s-s_{1}\right)\left(s-s_{3}\right)}{\left(s_{2}-s_{1}\right)\left(s_{2}-s_{3}\right)}$

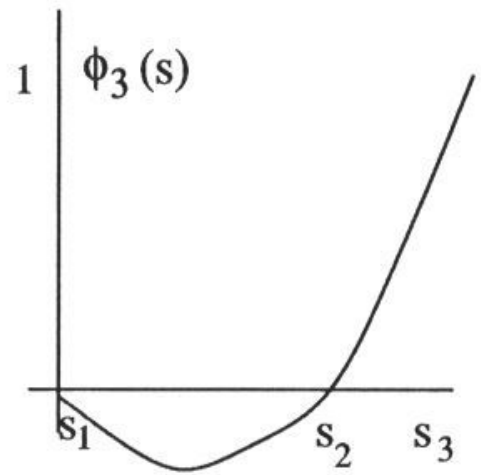

$\phi_{1}(s)=\frac{\left(s-s_{1}\right)\left(s-s_{2}\right)}{\left(s_{3}-s_{1}\right)\left(s_{3}-s_{2}\right)}$

Figure 5 Basis functions (Lagrange interpolating polynomials)

where $\Phi_{j}(s)(j=1, \ldots n)$ are the Lagrange interpolating polynomials (see Figure 5), and $x_{j}, y_{j}$ are the coordinates of the position vector $\mathrm{v}(\mathrm{s})$ at the nodes within the element.

A graphical representation of the basis functions is given in Figure 5. Note that several of the desirable computational properties of the final matrix are due to the use of the Lagrangian basis functions.

Calculation of the "element" equations. For each element we substitute the right hand side of equations (2) into expression (1) where the integration is taken over the corresponding element and we differentiate with respect to the unknowns, $x_{j}, y_{j}$, within each element. This leads to a system of $n$ equations in $n$ unknowns, the so-called element equations.

Derivation of the final system. The $\mathrm{m}$ sets of $\mathrm{n}$ element equations are then assembled to form the final system of $M$ equations in $M$ unknowns, by summing the coefficients at the inter-element boundaries. The resulting $M^{*} M$ matrix (the "stiffness" matrix) has symmetric, positive-definite characteristics, and a variety of numerical schemes exist that offer an efficient computational solution.

\section{(2) Modification for model-based vision}

The conventional FEM has been adapted to the specific needs of image processing in the following way.

The initial positions of the nodes (see Figure 3(top)) are determined interactively by hand, and are identified as fixed or movable points. The nodes are grouped into elements consisting of three nodes, so that neighbouring elements share their boundary nodes (see Figure 4(b)). The positions of the nodes determines the forces exerted on them by the internal tension of the model. These internal forces are combined into a global stiffness matrix, $\mathbf{A}$.

The stiffness matrix $\mathbf{A}$, representing the internal forces of the snake, is combined with the external forces, which cause the snake to be attracted towards areas of low intensity in the image. This leads to equation (3).

$$
\mathbf{A} * \mathbf{v}=\mathbf{b}
$$

Where $\underline{b}$ represents the force vector due to the image energy term. The force vector is derived numerically from the image data by measuring the change, under small perturbations of the position $\mathrm{v}(\mathrm{s})$, in the integral of $\mathrm{I}(\mathrm{v}(\mathrm{s})$ ) along lines between adjacent nodes within an element.

Equation (3) is then modified to take account of the boundary conditions due to fixed nodes by the method given in [12, page 173].

The external force vector $\underline{b}$ depend strongly on the position of the model in the image, so equation (3) is non-linear, and a simple iterative scheme is employed to solve it. At each iteration, an improved position for $\mathrm{v}(\mathrm{s})$ is computed by the linear solution of (3). This provides an improved estimate for $\underline{b}$, and the process is repeated. At each step, a node is constrained to move no further than a fixed limit, which is progressively reduced with each iteration. This has the effect of damping down any oscillatory behaviour.

\section{References:}

1. Roberts. L.G. Machine Perception of 3-d solids. In: Optical and Electro-optical Information Processing, J.P. Tippett, et al (Eds), MIT Press. 1965.

2. Brooks, R.A. Symbolic Reasoning among 3-d Models and 2-d Images. Stanford AI-Lab Memo AIM343, 1981.

3. Kass, M., Witkin, A., and Terzopoulos D. Snakes: Active Contour Models, Int. J. Computer Vision 1. 321 - 331, 1987.

4. Terzopoulos, D., Witkin, A. and Kass, M. Symmetry-seeking models and 3D object reconstruction, Int. J. Comp Vis. 1, 211-221. 1987.

5. Terzopoulos D., Witkin A and Kass M. Constraints on Deformable Models: Recovering 3D shape and Nonrigid Motion. AI, 36, 91-123, 1988. 
6. Arthurs, A.M.: Complementary Variational Principles, Oxford Math. Monographs, 1970

7. Burnett, D. S.: Finite Element Analysis, Addison Wesley, 1987.

8. Gelfand M. and Fomin S. Calculus of Variations, Prentice-Hall, 1963

9. Reddy, J.N. Applied Functional Analysis and Variational Methods in Engineering. McGraw-Hill, 1986.

10. Weaver, W. and Johnston, R.P. Finite Elements for Structural Analysis. Prentice Hall, 1984.

11. Press, H.W., Flannery, P.B. Teukolsky, A.S. and Vetterling, T.W. Numerical Recipes, Revised Edition, CUP, 1988.

12. Reddy, J.N.: An introduction to the Finite Element Method, McGraw-Hill, 1984 